\title{
HIGH RESOLUTION THRESHOLD PHOTODETACHMENT SPECTROSCOPY OF NEGATIVE IONS
}

\author{
T.N. KITSOPOULOS, I.M. WALLER ${ }^{1}$, J.G. LOESER ${ }^{2}$ and D.M. NEUMARK ${ }^{3}$ \\ Department of Chemistry, University of California, Berkeley, CA 94720, USA
}

Received 15 May 1989

\begin{abstract}
We have designed and constructed a negative ion threshold photodetachment spectrometer with $3 \mathrm{~cm}^{-1}(0.37 \mathrm{meV})$ resolution. Threshold photodetachment spectra of $\mathrm{I}^{-}$and $\mathrm{SH}^{-}$have been obtained. For $\mathrm{SH}^{-}$, individual rotational transitions between the ion and neutral appear as clearly resolved peaks.
\end{abstract}

\section{Introduction}

In recent years, negative ion photoelectron spectroscopy (PES) has emerged as a versatile technique well-suited to the study of a variety of exotic chemical species. These species include reactive free radicals [1], size-selected clusters [2], and the unstable collision complexes formed in chemical reactions [3]. In a typical experiment, a mass-selected negative ion beam is photodetached with a fixed-frequency laser, and some fraction of the ejected photoelectrons is collected and energy-analyzed. The experiment can be used to study the vibrational and electronic spectroscopy of both the negative ion and the neutral resulting from photodetachment, and, for example, can probe electronic states of the neutral which are inaccessible to more conventional optical spectroscopic methods $[1,4]$.

A significant drawback to this technique is the relatively poor (compared to optical spectroscopy) resolution of the electron energy analyzer. The energydispersive and time-of-flight analyzers used in fixed frequency PES generally have a resolution limit of 5-10 meV. This is sufficient to observe some vibration and electronic structure in small molecules. However, for many systems of interest, particularly clusters of moderate size, this limit prevents the ob-

\footnotetext{
I NSERC (Canada) Postdoctoral Fellow.

2 NSF Predoctoral Fellow.

3 NSF Presidential Young Investigator.
}

servation of vibrational features in the spectrum.

We have constructed a threshold photodetachment spectrometer which combines the advantages of mass-selected negative ion photodetachment with a high resolution threshold photoelectron detection scheme developed by Schlag and co-workers [5] for the study of neutral photoionization. In this scheme, a pulsed tunable laser is used as the photoionizing (or photodetaching) light source, and a weak extraction field is applied to the interaction region a short time after the laser fires. This allows the selective detection of the near-zero kinetic energy electrons produced at the laser frequency. These slow electrons will only be produced when the photon energy is at or just above the threshold for an ion $\rightarrow$ neutral transition. If the electron detection sys. tem counts only those photoelectrons produced with a center-of-mass kinetic energy less than $\Delta E$, the spectrum of the threshold photoelectron signal as a function of photon energy should exhibit a peak of width $\Delta E$ at each ion $\rightarrow$ neutral transition. In principle, the same information is available from fixedfrequency photoelectron spectroscopy, but the resolution in a threshold photoelectron spectrum can be much higher. In our adaptation of this method, we have achieved a resolution of $0.37 \mathrm{meV}\left(3 \mathrm{~cm}^{-1}\right)$.

In this paper, we demonstrate the performance of this instrument by presenting the threshold photodetachment spectra of $\mathrm{I}^{--}$and $\mathrm{SH}^{-}$. In the latter spectrum, individual ion $\rightarrow$ neutral rotational transitions are resolved as peaks in the spectrum; in effect, 
we have obtained the rotational resolved photoelectron spectrum of a negative ion.

\section{Experimental}

A schematic of the apparatus is presented in fig. 1. Negative ions formed in a free jet expansion $(1,2)$ pass through a skimmer and are accelerated to $500 \mathrm{eV}$ with the ion lens stack (3). Mass-selection is achieved with a coaxial time-of-flight arrangement consisting of two beam modulation plates (4), an Einzel lens (5), a second pulsed field applied at the plates (6), and a defining slit (7). The pulsed laser intersects ions of the desired mass between the first and second plate of the lens stack (8). The remaining ions or neutral products formed by photodetachment are detected at $(10)$. The threshold photoelectrons are extracted by the lens stack ( 8 ), focused by the Einzel lens (9), and deflected into the electron detector (11).

The apparatus consists of an ion source chamber, two differently pumped regions, and a detector chamber. The ion source and two differential regions are pumped by $10^{\prime \prime}, 6^{\prime \prime}$, and 2" Edwards Diffstak diffusion pumps, respectively. The detector chamber is pumped by an Alcatel $450 \mathrm{\ell} / \mathrm{s}$ turbomolecular pump. The skimmer ( $2 \mathrm{~mm}$ diameter, Beam Dynamics) separates the ion source from the first differential region. The first and second regions are separated by a $5 \mathrm{~mm}$ diameter slit which is part of the first element in the Einzel lens (8). The $5 \mathrm{~mm}$ diameter mass spectrometer defining slit (7) separates the detector from the second differential region. Typical operating pressures in the four regions (from

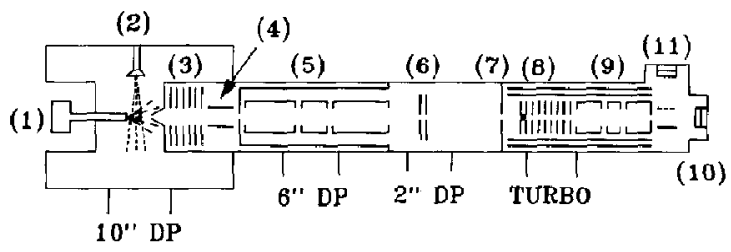

Fig. 1. Schematic of apparatus: (1) Pulsed molecular beam valve. (2) Electron gun. (3) Ion lens stack. (4) Beam modulation plates.

(5) Ion einzel lens. (6) Temporal focusing plates. (7) $5 \mathrm{~mm}$ slit.

(8) Electron extraction plates. (9) Electron einzel lens. (10) Ion/ neûtral detector. (11) Electron detector. source to detector) are $4 \times 10^{-5}, 2 \times 10^{-6}, 5 \times 10^{-7}$ and $1 \times 10^{-8}$ Torr.

\subsection{Ion source and mass spectrometer}

Negative ions are generated using the pulsed molecular beam/electron gun configuration developed by Lincbcrger and co-workers [6]. Dilute mixtures of reagent gases are expanded through a pulsed molecular beam valve (Newport or General Valve) operated at a $50 \mathrm{~Hz}$ repetition rate. A $5 \% \mathrm{CF}_{3} \mathrm{I} / \mathrm{He} \mathrm{mix}$ is used for $\mathrm{I}^{-}$, and a $5 \% \mathrm{H}_{2} \mathrm{~S} / 15 \% \mathrm{NF}_{3} / \mathrm{He} \operatorname{mix}$ is used for $\mathrm{SH}^{-}$. The valve orifice diameter is $0.030^{\prime \prime}$, and typical backing pressures range from 20 to 40 psig. A few mm downstream from the valve orifice, the molecular beam is crossed with a $1 \mathrm{keV}, 250 \mu \mathrm{A}$ electron beam from a Tektronix electron gun. The slow secondary electrons resulting from the ionization by the $1 \mathrm{keV}$ electrons efficiently produce $\mathrm{I}^{-}$ from $\mathrm{CF}_{3} \mathrm{I}$ and $\mathrm{F}^{-}$from $\mathrm{NF}_{3}$ by dissociative attachment; $\mathrm{SH}^{-}$is subsequently formed by the reaction $\mathrm{F}^{-}+\mathrm{H}_{2} \mathrm{~S} \rightarrow \mathrm{SH}^{-}+\mathrm{HF}$. Since the ions are formed in the continuum flow region of the free jet expansion, they are expected to internally cool as the expansion progresses. This is confirmed by our $\mathrm{SH}^{-}$results (see below). The skimmer lies about $2.5 \mathrm{~cm}$ downstream from the valve orifice. The electron gun, beam valve, skimmer, and surrounding electrostatic shielding are all floated at $-500 \mathrm{~V}$ with respect 10 ground.

In the first differential region, $2 \mathrm{~cm}$ downstream from the skimmer base, a stack of nine ring electrodes (3) accelerates the ions coaxially to $500 \mathrm{eV}$. The voltage drops geometrically from $-500 \mathrm{~V}$ to ground across the stack.

The accelerated ions are mass-selected with a beam-modulated time-of-flight mass spectrometer based on the design by Bakker [7]. The essential components of the mass spectrometer are the (square) bcam modulation plates (4), which arc 6 $\mathrm{cm}$ on a side and separated by $1 \mathrm{~cm}$, and the $5 \mathrm{~mm}$ diameter defining slit (7) which lies $100 \mathrm{~cm}$ downstream from the plates. One plate is held at voltage $\frac{1}{2} V$, and the other is rapidly switched ( 15 ns fall time) from $V$ to 0 several hundred $\mu$ s after the beam valve fires. $V$ is typically $20 \mathrm{~V}$. Only the ions in a small volume midway though the plates when the voltage is switched pass through the defining slit. These ions separate into bunches according to their masses. The 
cylindrical Einzel lens (7), $7.5 \mathrm{~cm}$ outer diameter, focuses the ion bunch perpendicularly to the beam direction. This configuration results in a mass resolution of 150-200.

To improve the temporal overlap of the selected ion bunch with the pulsed laser beam, a longitudinal pulsed acceleration field is applied using the two annular plates (6), which are located $15 \mathrm{~cm}$ in front of the defining slit. The plate closest to the defining slit is grounded while the other ( $2.5 \mathrm{~cm}$ away) is pulsed to between -10 and $-20 \mathrm{~V}$. The pulse is applied when the ion bunch of interest is located between the two plates. This focuses the ions along the beam axis. The exact position of this focus depends on the strength of the pulsing field. At the ion detector, the width of the $\mathrm{I}^{-}$peak (at $70 \mu$ s flight time) is reduced from 300 ns to $<100$ ns using this temporal focusing.

The ion signal is monitored by a Chevron-mounted pair of $25 \mathrm{~mm}$ microchannel plates (Galileo) located at the end of the drift tube. Alternatively, by negatively biasing a grid in front of this detector the ions are rejected but the neutrals generated upon photodetachment of the ion beam can be monitored.

The ions of interest are photodetached using an excimer pumped tunable dye laser (Lambda Physik EMG 202 and FL 3002). The light is introduced at $90^{\circ}$ to the ion beam axis and a small fraction of the power is monitored using a pyroelectric detector (Molectron P1-61). Laser dyes were obtained from Exciton; DPS (399-415 nm) was used for the $\mathrm{I}^{-}$ spectra, and Coumarin 540A (522-600 nm) was used to obtain the $\mathrm{SH}^{-}$spectra.

\section{2. Threshold electron detection}

Various methods for the selective detection of threshold photoelectrons have been developed during the last several years $[8,9]$. In all of these approaches, electrons are extracted from the interaction region with a weak, uniform electric field. A set of apertures spatially discriminates against those energetic electrons with a substantial velocity component perpendicular to the field direction. If a pulsed light source is used, time-gated detection can be used to temporally discriminate against energetic electrons ejected parallel or antiparallel to the field. Miiller-Dethlefs et al. [5] showed that with a pulsed light source, both spatial and temporal discrimina- tion were dramatically improved by introducing a delay on the order of $1 \mu$ s between the light pulse and application of the extraction field. This yielded photoionization threshold peaks $1 \mathrm{~cm}^{-1}$ wide, about a factor of ten improvement over the best result obtained with a cw light source [9].

Our implementation of this method is constrained since we are photodetaching a fast $(500 \mathrm{eV})$ ion beam instead of nearly stationary neutral molecules. In our experiment, threshold electrons formed from $\mathrm{SH}^{-}$will have $8 \mathrm{meV}$ of kinetic energy in the lab frame of reference and a velocity of $5.4 \times 10^{6} \mathrm{~cm} / \mathrm{s}$ along the beam axis. These electrons will travel 5.4 . $\mathrm{cm}$ during the $1 \mu \mathrm{s}$ delay. Hence, an extended coaxial extraction field must be used.

We use a parallel array of 16 molybdenum round plates, $0.030^{\prime \prime}$ thick, $2.5^{\prime \prime}$ outer diameter, and $0.5^{\prime \prime}$ inner diameter, mounted symmetrically about the instrument axis (8). The first four plates are separated by $10 \mathrm{~mm}$. These are connected by $10 \Omega$ resistors so that they produce a uniform electric field parallel to the instrument axis. The first plate is pulsed between -5 and $-10 \mathrm{~V}$ while the fourth and successive plates, separated by $5 \mathrm{~mm}$, are grounded. The latter plates act as a series of apertures. The ions are photodetached between the first and second plates.

After the last plate, there is a $60 \mathrm{~cm}$ drift region. To improve the threshold electron collection efficiency, this region contains a 3 " outer diameter cylindrical decelerating einzel lens made from OFHC copper (9). A deflection field $(5 \mathrm{~V} / \mathrm{cm})$ is located immediately after the drift region and is used to deflect the photoelectrons onto the electron detector (11), a Chevron-mounted pair of $25 \mathrm{~mm}$ microchannel plates mounted perpendicular to the beam axis.

A delay (250-500 ns) is introduced after photodetachment with the pulsed laser and before application of the extraction field. During this time energetic electrons which have perpendicular velocity components will move far enough off axis so that they can be geometrically discriminated against by the apertures. Only the electrons which remain on axis are accelerated when the extraction field is applied. This includes the backward and forward ejected energetic electrons as well as the threshold (zero energy) electrons. These three groups of electrons have 
spatially separated during the time delay; thus they travel different distances in the field and gain different amounts of energy. In fact, the electrons scattered backward prior to extraction spend the most time in the field and so gain the most energy from it. Thus the flight time to the electron detector is different for the threshold electrons than for the more cnergetic coaxially ejceted elcctrons. The threshold electrons can be selectively detected within a 50-100 ns time window.

All photoelectron optics mentioned here are mounted inside two coaxial tubes made from $\mathrm{Hy}$ pernom. These tubes provide magnetic shielding from the earth's magnetic field and are essential to the experiment.

The electron signal is preamplified (Ortec 9301) and integrated by a LeCroy 2249 SG gated A/D converter and stored in an AT computer after each laser shot. The ion signal and laser power signal are integrated with the LeCroy 2249SG and stored for normalization purposes. All timing is controlled with a Stanford Research Systems DG535 delay generator.

In a typical experiment, we signal average for 200 500 laser shots at each frequency and detect $0.5-5$ threshold electrons per shot. The ion density resulting in this signal lcvel is cstimated to be $10^{4} \mathrm{~cm}^{-1}$. At higher ion densities, space charge interactions between the electrons and the ion cloud become noticeable. It is likely that even at the ion densities used in this work, space charge effects are the dominant broadening mechanism in the spectra. The problem of photoelectron interaction with an ion cloud is far more severe in negative ion photodetachment than in neutral photoionization.

\section{Results and discussion}

\subsection{General considerations}

The energy dependence of the photodetachment cross section for a negative ion $\rightarrow$ neutral transition is given by $\sigma \propto\left(E-E_{\text {th }}\right)^{l+1 / 2}$, where $E_{\text {th }}$ is the threshold energy for the transition and $l$ is the orbital angular momentum of the ejected electron [10]. The cross section will be large within a few $\mathrm{cm}^{-1}$ of threshold only if s-wave $(l=0)$ photodetachment occurs. Because $l=0$ electrons do not experience a cen- trifugal barrier, s-wave photodetachment generally dominates near a threshold unless forbidden by symmetry; the necessary conditions for an s-wave have been discussed by Reed et al. [11]. The two ions reported here, $\mathrm{I}^{-}$and $\mathrm{SH}^{-}$, are known from previous wrk to undergo s-wave photodetachment $[12,13]$. These considerations are not a problem in neutral photoionization for which the cross section is a step function at each threshold.

An advantage of our experiment compared to threshold photoionization of neutrals is that since negative ions do not have Rydberg states, we generally do not have to worry about either field ionization of states lying below a threshold or the production of slow electrons by autoionizing states. Negative ions with excited electronic or vibrational states lying near the detachment threshold are the exception rather than the rule [14].

\subsection{Photodetachment of $\mathrm{I}^{-}$}

$\mathrm{I}^{-}$was chosen as the first test of the instrument. The electron binding energy of $\mathrm{I}^{-}$was previously determined to be $3.0591 \mathrm{eV}(405.3 \mathrm{~nm})$ by laser optogalvanic spectroscopy [13].

In fig. 2, the threshold photoelectron spectrum we obtain for the photodetachment of $\mathrm{I}^{-}$is presented. The peak in the spectrum is from the transition

$I^{-}\left({ }^{1} S_{0}\right)+h \nu \rightarrow I\left({ }^{2} P_{3 / 2}\right)+\mathrm{e}^{-}$.

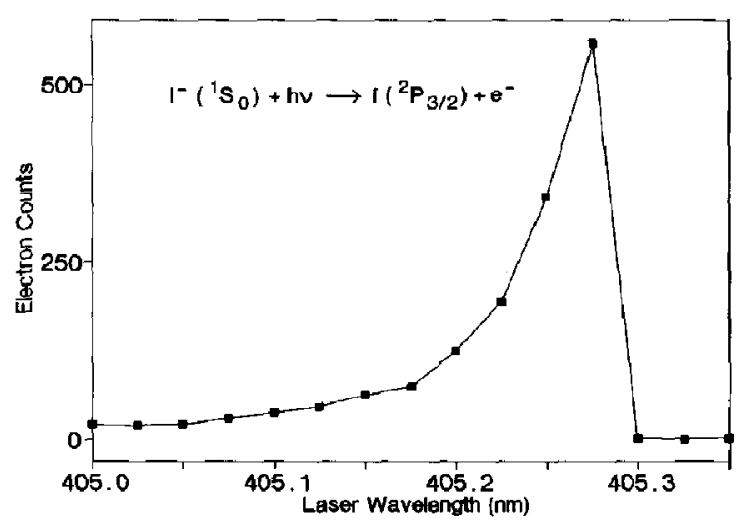

Fig. 2. Threshold photodetachment spectrum of $\mathrm{I}^{-}$. The (fwhm) width is $3 \mathrm{~cm}^{-1}$. Each point represents the total number of electrons collected for 200 laser pulses. The DPS laser dye output was approximately $10 \mathrm{~mJ} /$ pulse. 
Data were taken at $0.025 \mathrm{~nm}$ intervals. The (fwhm) width of the peak is $3 \mathrm{~cm}^{-1}$. The peak rises sharply at the threshold for this transition and falls off more slowly to lower wavelength. This "saw-tooth" peak shape results from discrimination against high kinetic energy electrons and is typical of all thresholds observed with our instrument.

\subsection{Photodetachment of $\mathrm{SH}^{-}$}

As a more interesting test of the capabilities of our instrument, we have measured the threshold photodetachment spectrum of $\mathrm{SH}^{-}$. Both $\mathrm{SH}$ and its anion have been well-characterized [15,16]. The neutral and anion have similar bond lengths, indicating that a $\Delta v=0$ transition will occur upon photodetachment of the anion to the ground state of SH. Since the $B_{0}$ values for hoth neutral and anion are fairly large, approximately $9.4 \mathrm{~cm}^{-1}$, it should be possible to resolve individual rotational transitions with our spectrometer.

The absolute photodetachment cross-section for $\mathrm{SH}^{-}$was first measured by Steiner [17]. Higher resolution measurements in an ion cyclotron resonance cell by Janousek and Brauman [18] and, more recently, by Larsen [19] showed rotational transitions appearing as changes in slope of the cross section. Breyer et al. [20] studied $\mathrm{SH}^{-}$with fixed-frequency photoelectron spectroscopy. At a resolution of 5-7 $\mathrm{meV}$, they were able to observe rotational contours but not individual rotational transitions.

In fig. 3 a rotational energy level diagram for the $\mathrm{SH}^{-}$, $\mathrm{SH}$ system is presented. Since the ejected electron has spin $1 / 2$, the selection rules for each ion $\rightarrow$ neutral transition are $\Delta J= \pm 1 / 2, \pm 3 / 2$, and there are a maximum of four possible transitions from a given rotational level in the anion to each spin-orbit state of the neutral [18,20]. For similar rotational constants in the ion and neutral, transitions in the $\Delta J= \pm 1 / 2$ branches should be approximately separated by $B$, and those in the $\Delta J= \pm 3 / 2$ branches by $3 B$.

Figs. $4 \mathrm{a}$ and $4 \mathrm{~b}$ show threshold spectra for transitions to the $\mathrm{SH}\left({ }^{2} \Pi_{3 / 2}\right)$ ground state and $\mathrm{SH}\left({ }^{2} \Pi_{1 / 2}\right)$ excited spin-orbit multiplet, respectively. Individual rotational transitions are resolved, and the peak assignments are indicated in the figure. This is the first observation of clearly resolved rotational peaks in

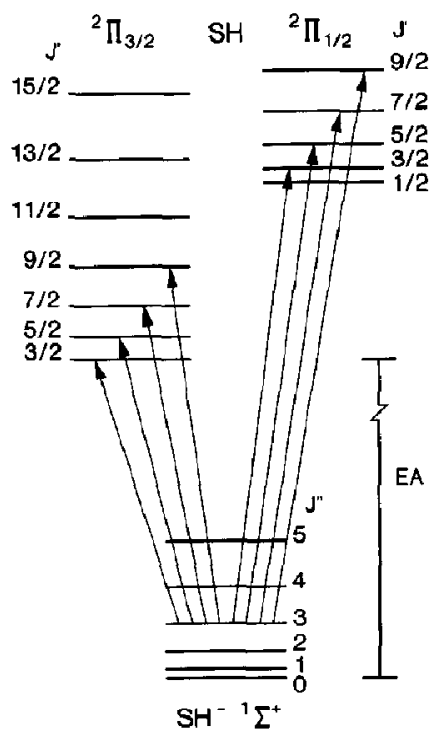

Fig. 3. Rotational energy level diagram for $\mathrm{SH}^{-}, \mathrm{SH}$ system. The allowed photodetachment transitions from $\mathrm{SH}^{-}\left(\mathrm{J}^{\prime \prime}=3\right)$ to the ground and spin-orbit excited state of $\mathrm{SH}$ are indicated. Four branches to each spin-orbit state are allowed, see text for explanation. The electron affinity of SH is $2.317 \pm 0.002 \mathrm{eV}$.

the direct photodetachment of a negative ion.

The intensities in both branches may be modelled fairly well by assuming a rotational Boltzmann temperature of $50-75 \mathrm{~K}_{\text {for }} \mathrm{SH}^{-}$. However, the distribution is not strictly Boltzmann, the transitions originating from $\mathrm{SH}^{-} J=0$ are relatively more intense than predicted by this temperature. In addition, higher rotational levels of $\mathrm{SH}^{-}$are likely to relax less efficiently in a free jet expansion. The apparent temperature is very sensitive to the expansion conditions. Backing pressure, gas pulse duration, the position where the electron beam intersects the molecular beam, and the relative timing of switching the beam modulation plates are all important factors. In any case, we are certainly obtaining substantially cooler ions than any other method used to study $\mathrm{SH}^{-}$to date. Several recent studies of ions and ion clusters have used an ion source similar to the one used here. Our results demonstrate that one can in fact obtain cold ions from this type of source, even after skimming the beam and subsequent acceleration fairly close to the beam valve. 

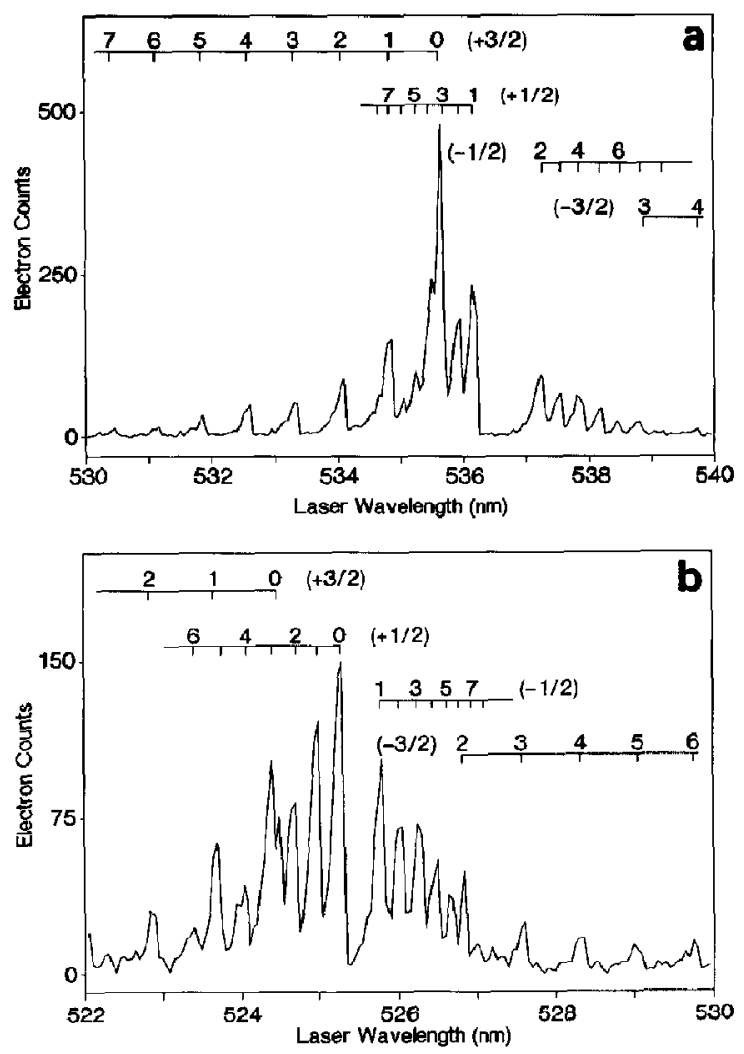

Fig. 4. (a) Threshold photodetachment spectrum for $\mathrm{SH}^{-}\left({ }^{1} \Sigma^{+}\right)+h \nu \rightarrow \mathrm{SH}\left({ }^{2} \Pi_{3 / 2}\right)+e^{-}$. The $\Delta J$ transitions for each branch are given in parentheses. The $\mathrm{SH}^{-} J^{\prime \prime}$ values are indicated. Each point represents the total number of electrons collected for 500 laser pulses. The peaks have a fwhm of approximately $4 \mathrm{~cm}^{-1}$. (b) Threshold photodetachment spectrum for $\mathrm{SH}^{-}\left({ }^{1} \Sigma^{+}\right)+h \nu \rightarrow \mathrm{SH}\left({ }^{2} \Pi_{1 / 2}\right)+\mathrm{e}^{-}$.

\section{Summary}

We have demonstrated the operation of a threshold photodetachment spectrometer with $3 \mathrm{~cm}^{-1}$ resolution. This instrument has been used to obtain a rotationally resolved photoelectron spectrum of a negative ion, $\mathrm{SH}^{-}$. We plan to apply this instrument to the study of a variety of problems, including the study of transition states via negative ion photodetachment and the spectroscopy of mass-selected metal and semiconductor clusters.

\section{Acknowledgement}

Support for this work from the Office of Naval Research Young Investigator Program under Grant No. N0014-87-K-0495 is gratefully acknowledged.

\section{References}

[1 ] H.B. Ellis and G.B. Ellison Jr., J. Chem. Phys. 78 ( 1983) 6541;

D.G. Leopold, K.K. Murray and W.C. Lineberger, J. Chem. Phys. 81 (1984) 1048;

R.D. Mead, A.E. Stevens and W.C. Lineberger, in: Gas phase ion chemistry, Vol, 3, ed, M.T. Bowers (Academic Press, New York, 1984) p. 213.

[2] J.V. Coe, J.T. Snodgrass, C.D. Friedhoff, K.M. McHugh and K.H. Bowen, Chem. Phys. Letters 124 (1986) 274;

D.G. Leopold, J. Ho and W.C. Lineberger, J. Chem. Phys. 86 (1987) 1715;

O. Chesnovsky, S.H. Yang, C.L. Pettiette, M.J. Craycraft, Y. Liu and R.E. Smalley, Chem. Phys. Letters 138 (1987) 119 ;

K.J. Taylor, C.L. Pettiette, M.J. Craycraft, O. Chesnovsky and R.E. Smalley, Chem. Phys. Letters 152 (1988) 347;

M.J. DeLuca, B. Niu and M.A. Johnson, J. Chem. Phys. 88 (1988) 5857.

[3] R.B. Metz, T.N. Kitsopoulos, A. Weaver and D.M. Neumark, J. Chem. Phys. 88 (1988) 1463;

A. Weaver, R.B. Metz, S.E. Bradforth and D.M. Neumark, J. Phys. Chem. 92 (1988) 5558.

[4] A. Weaver, R.B. Metz, S.E. Bradforth and D.M. Neumark, J. Chem. Phys. 90 (1989) 2070.

[5]K. Müller-Dethlefs, M. Sander and E.W. Schlag, Z. Naturforsch. 39a (1984) 1089;

K. Müller-Dethlefs, M. Sander and E.W. Schlag, Chem. Phys. Letters 12 (1984) 291;

M. Sander, L.A. Chewter, K. Müller-Dethlefs and E.W. Schlag, Phys. Rev. A 36 (1987) 4543.

[6] M.A. Johnson, M.L. Alexander, I. Hertel and W.C. Lineberger, Chem. Phys. Letters 12 (1984) 285.

[7] J.M.B. Bakker, J. Phys. E 6 (1973) 785; 7 (1974) 364.

[8] T. Baer, W.B. Peatman and E.W. Schlag, Chem. Phys. Letters 4 (1969) 243;

R. Spohr, P.M. Guyon, W.A. Chupka and J. Berkowitz, Rev. Sci. Instr. 42 (1971) 1872;

R. Frey, B. Gotcher, O.F. Kalman, W.B. Peatman, H. Pollak and E.W. Schlag, Chem. Phys. 21 (1977) 89.

[9] W.B. Peatman, G.B. Kasting and D.J. Wilson, J. Electron Spectry. 7 (1975) 233.

[10] E.P. Wigner, Phys. Rev. 73 (1948) 1003.

[11] K.J. Reed, A.H. Zimmerman, H.C. Anderson and J.I. Brauman, J. Chem. Phys. 64 (1976) 1368.

[12] C.R. Webster, I.S. McDermid and C.T. Rettner, J. Chem. Phys. 78 (1983) 646.

[13] T.E.H. Walker, Chem. Phys. Letters 19 (1973) 493. 
[14] D.M. Neumark, K.R. Lykke, T. Andersen and W.C. Lineberger, J. Chem. Phys. 83 (1985) 4364;

R.D. Mead, K.R. Lykke, W.C. Lineberger, J. Marks and J.I. Brauman, J. Chem. Phys. 81 (1984) 4883.

[15] P.F. Bernath, T. Amano and M. Wang, J. Mol. Spectry. 98 (1983) 20.

[16] M. Gruebele, M. Polak and R.J. Saykally, J, Chem, Phys. 86 ( 1987$) 1698$.
[17] B. Steiner, J. Chem. Phys. 49 (1968) 5097.

[18] B.K. Janousek and J.I. Brauman, Phys. Rev. A 23 (1981) 1673.

[19] D.J. Larson, C.J. Edge, R.E. Elmquist, N.B. Mansour and R. Trainham, Physica Scripta T 22 (1988) 183.

[20] F. Breyer, P. Frey and H. Hotop, Z. Physik A 300 (1981) 7. 